\title{
Acellular Synthesis of a Human Enamel-like Microstructure**
}

\author{
By Haifeng Chen, Zhiyong Tang, Jun Liu, Kai Sun, Sywe-Ren Chang, Mathilde C. Peters, \\ John F. Mansfield, Agata Czajka-Jakubowska, and Brian H. Clarkson*
}

Dental enamel is the outermost layer of teeth and the hardest mineralized tissue in the human body. ${ }^{[1,2]}$ It consists of nanorod-like hydroxyapatite (HA) crystals arranged into a highly organized micro-architectural unit called an enamel prism. These special units play an important role in determining the unique physicochemical properties of dental enamel. Cells (ameloblasts) and enamel proteins are thought to be intimately involved in vivo in producing this unique structure. The aim of this research was to create a similar structure directly by crystal growth without using cells and/or enamel proteins. Using a hydrothermal method we have been able to synthesize these prism-like structures, consisting of fluorapatite (FA) crystals, which are similar to the dimensions of those seen in human enamel. Dental pulp stem cells (DPSCs) were cultured on these crystals and showed the excellent biocompatibility of the FA crystals. The growth mechanism of this structure is outlined and its use in tissue repair is discussed.

Ninety-five percent (by volume) of human enamel is comprised of nanorod-like calcium hydroxyapatite crystals, which have an approximate cross section of 25-100 nm and an undetermined length of $100 \mathrm{~nm}$ to $100 \mu \mathrm{m}$ or longer along the $c$-axis. ${ }^{[1-5]}$ The typical human enamel prism structure is approximately $5 \mu \mathrm{m}$ in cross section, and can span the entire enamel thickness, that is, approximately $1-2 \mathrm{~mm}$ in length. ${ }^{[-4]}$ The prevailing theory is that the ameloblasts secrete amelogenin, a major enamel protein constituting approximately

[*] Prof. B. H. Clarkson, Dr. H. Chen, Dr. J. Liu, S.-R. Chang, Prof. M. C. Peters

School of Dentistry, University of Michigan

1011 North University, Ann Arbor, MI 48109-1078 (USA)

E-mail: bricla@umich.edu

Dr. Z. Tang

Department of Chemical Engineering, University of Michigan

2300 Hayward, Ann Arbor, MI 48109-2176 (USA)

Dr. K. Sun, Dr. J. F. Mansfield

Electron Microbeam Analysis Laboratory, University of Michigan 2455 Hayward, Ann Arbor, MI 48109-2143 (USA)

Dr. A. Czajka-Jakubowska

Karol Marcinkowski University of Medical Sciences

Department of Conservative Dentistry and Periodontology Poznan (Poland)

[*** This investigation was supported by a USPHS Research Grant DE015599 from the National Institute of Dental and Craniofacial Research, National Institutes of Health, Bethesda, MD 20892. The JEOL-2010F SEM/TEM used for this investigation was funded by a National Science Foundation Grant DMR-9871177. Supporting information is available online from Wiley InterScience or from the author.
$90 \%$ of all organic matrix material in developing enamel, and this protein plays a vital role in enabling crystallites to form a well-organized prism pattern. ${ }^{[6-8]}$ These prisms project from the dentino-enamel junction to the enamel surface. The prisms are aligned parallel to each other and separated by an interprismatic structure to form a densely compacted enamel layer. $^{[1,2]}$ Unlike other calcified tissues, such as dentin and bone, there are no living cells in the mature enamel. The ameloblast cells die after the enamel is formed. Thus, when the enamel is damaged, the body has no ability to regenerate it.

The approach to create artificial bone and tooth structures has attracted the interest of many researchers. ${ }^{[4,9-14]}$ Kniep and co-workers have described a fluorapatite-gelatine system that resembles the biosystem hydroxyapatite-collagen in both bone and dentine. ${ }^{[13]}$ Recently, Yamagishi et al. have reported a paste of fluoridated hydroxyapatite that could be used to repair a small carious lesion. Although the interface between the precipitated layer from the paste and the tooth surface contains elongated crystals some of which are orientated towards the tooth surface, the unique enamel prism structure is not visible from the transmission electron microscopy (TEM) images. ${ }^{[4]}$ In our previous paper, we reported a way to mimic the natural biomineralization process to create these special structures by modifying synthetic hydroxyapatite nanorods with surfactants that allow the nanorods to self-assemble into an enamel prismlike structure at a water/air interface. ${ }^{[14]}$ However, those self-assembled enamel prism-like structures are small, only about $400 \mathrm{~nm}$ in length and $100 \mathrm{~nm}$ in cross section, and dispersed randomly. Very recently, Fowler et al. reported that they were able to synthesize an HA bundle structure directly from a solution containing the surfactant bis(2-ethylhexyl)sulfosuccinate sodium salt (AOT), water, and oil. These bundles were only $750 \mathrm{~nm}$ to $1 \mu \mathrm{m}$ in length, 250-350 nm wide and they were dispersed randomly. ${ }^{[12]}$ These unique structures, therefore, have limited applications, and the introduction of other chemicals, for example AOT may cause unnecessary biological effects.

The development of nanotechnology has created many ways to grow $1 \mathrm{D}$ nanostructures. ${ }^{[15-17]}$ Among them, the hydrothermal method is a widely adopted technique to create nanorods, nanowires, and whiskers and has already been shown to be an effective way to create long hydroxyapatite nanorods and whiskers. ${ }^{[18-20]}$

In this study, we demonstrate a direct growth method to produce fluorapatite dental enamel prismlike structures using a hydrothermal technique. A film of compacted well-aligned FA crystals, which was grown on metal plates, had a structure 
very similar to human enamel. Using scanning electron microscopy (SEM), energy-dispersive X-ray spectroscopy (EDS), TEM, powder X-ray diffraction (XRD), and Fouriertransform infrared spectroscopy (FTIR), the mechanism of how these structures form was explored. DPSCs were cultured on the above crystals in order to demonstrate the biocompatibility of these FA crystals.

Figure 1 shows the SEM image of an etched human enamel surface and human enamel crystals. The prisms are approximately $5 \mu \mathrm{m}$ in cross section and made of bundles of nanorodlike calcium hydroxyapatite crystals arranged parallel to each other (Fig. 1a). The profile image shows that these crystals are approximately $80 \mathrm{~nm}$ in cross section and over $1 \mu \mathrm{m}$ in length along the $c$-axis (Fig. 1b).

The morphology of synthetic FA crystals varies depending on the autoclave time. In the early stage, while at room temperature, the suspensions consist of mainly amorphous particles and very few rodlike structures (see Fig. S1 in the Supporting Information for the TEM and EDS data of an early-stage deposit). However, after autoclaving for $5 \mathrm{~min}$, the amorphous deposit is almost totally replaced by ball-like structures and more rodlike structures become apparent (Fig. 2a-e). Some of these ball-like structures contain solid inclusions (Fig. $2 b$ and c). The TEM images show that the outer shell of the ball is made of many small crystals of different orientation (Fig. 2g), while the rodlike structures are made of many small crystals aligned parallel to each other (Fig. 2e) and have a typical apatite crystalline structure (Fig. 2f). The ball-like structures contain relatively more fluoride than the rodlike structures, which is similar to the EDS data shown in Figure S1f. After autoclaving for a longer time, only the rodlike structures are seen. Figure 3 shows the SEM images of samples after autoclaving for $10 \mathrm{~min}$ to $10 \mathrm{~h}$. As the autoclaving time increased, the crystals changed from shuttlelike structures (Fig. 3a and b) to well-defined microcrystals (Fig. $3 \mathrm{e}$ and $\mathrm{f}$ and Fig. 4a and b). The well-defined crystals with a typical apatite hexagonal structure were created after autoclaving for $10 \mathrm{~h}$ (Figs. 3f and 4b). The crystals are very well aligned over a large area after being deposited on an iron plate, as shown in Figure 4c and $\mathrm{d}$. The rods spontaneously form bundles (enamel prismlike structures) as shown in Figure $4 \mathrm{a}$ and $\mathrm{b}$ (crystals $1-3 \mu \mathrm{m}$ in cross section and approximately $50 \mu \mathrm{m}$ in length, cross section of prism structures 10-30 $\mu \mathrm{m}$ ). At lower power, the FA crystals that are densely deposited on an iron plate (Fig. 4c)

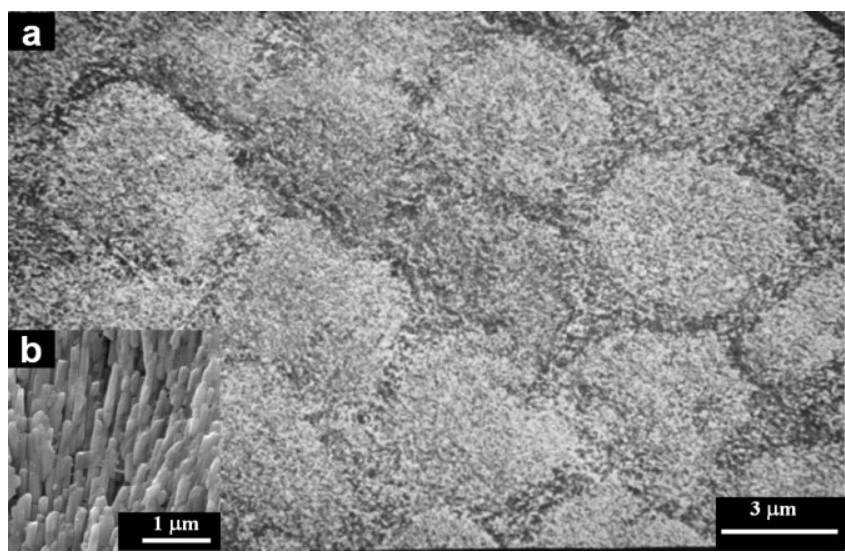

Figure 1. a) SEM image of the natural surface of human molar enamel which was lightly etched with $37 \%$ phosphoric acid; b) SEM image of human molar enamel which was cut longitudinally with a diamond saw.
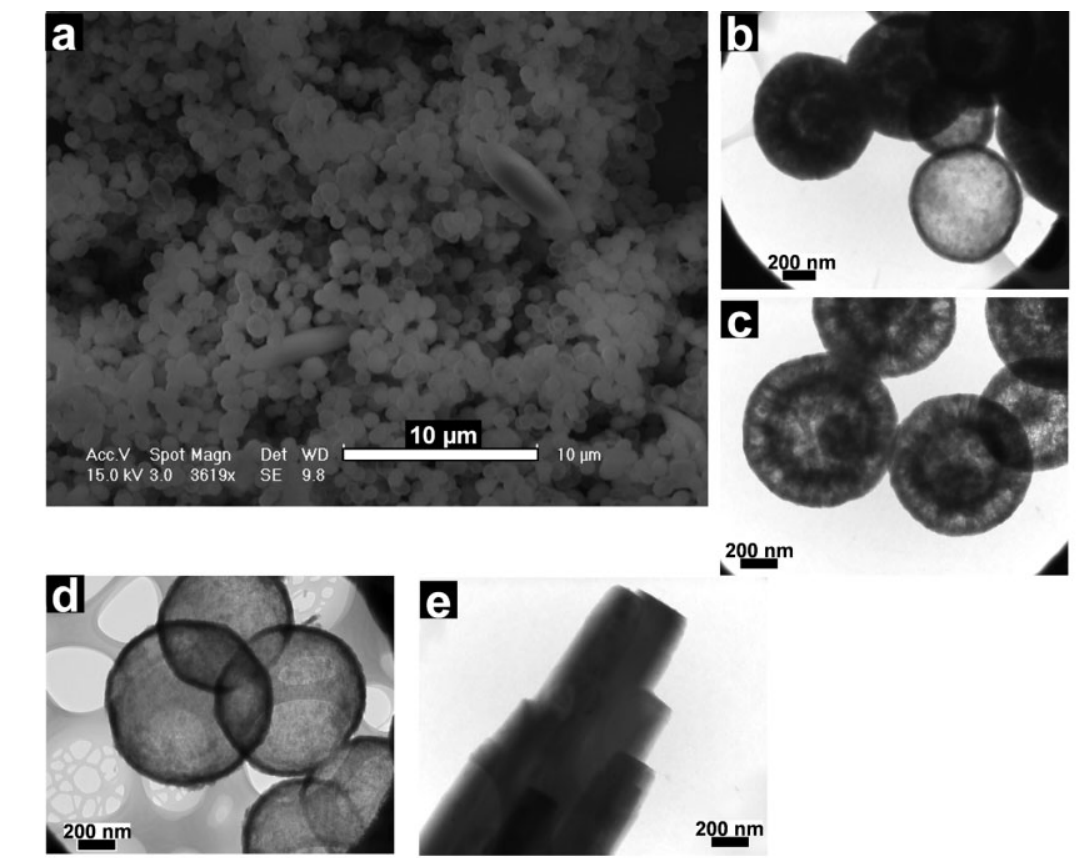

$200 \mathrm{~nm}$

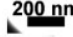

$200 \mathrm{~nm}$

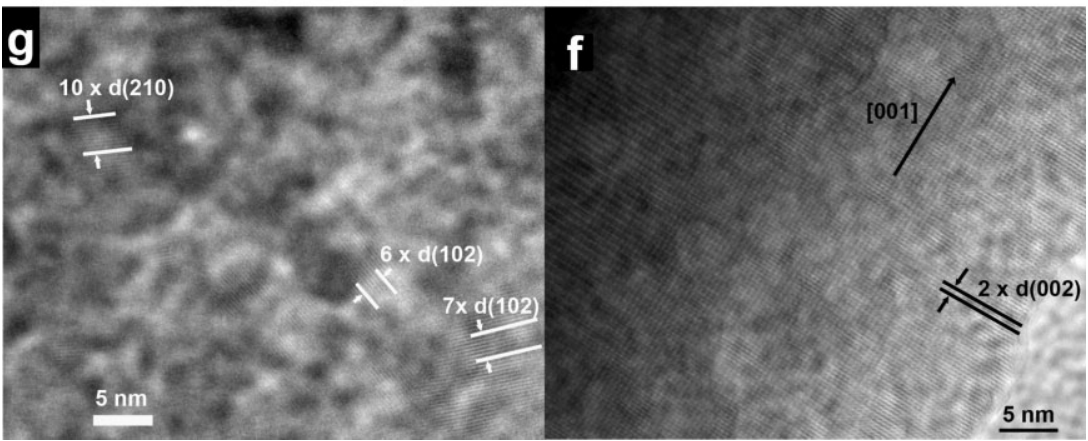

Figure 2. SEM and TEM images after autoclaving for $5 \mathrm{~min}$. a) This SEM image shows that the sample mainly consists of ball-like structures and some rodlike structures. b-d) TEM images show the ball-like structures at higher resolution. e) The TEM image shows part of the rod-like structures are made of small rods aligned parallel to each other. $f$ ) A high-resolution (HR)TEM image of rodlike structures showing the typical apatite characteristics. g) HRTEM image of the wall of a ball-like structure, which shows the nanocrystals contained within it. 

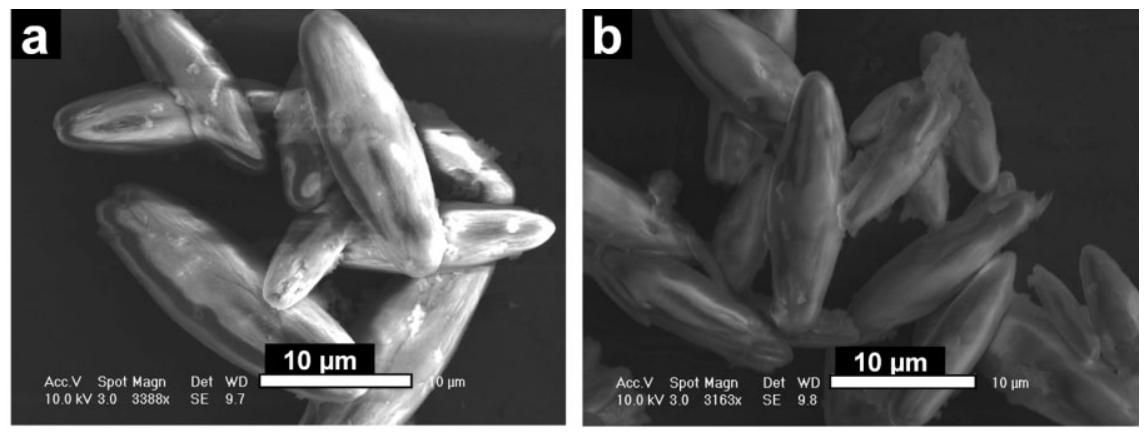

pure hexagonal phase with lattice constants $a=9.331 \AA$ and $c=6.840 \AA$, which is in accordance with FA. ${ }^{[21]}$ Also, evidence is presented demonstrating the biocompatibility of these crystals with dental pulp stem cells (see Fig. S5 in the Supporting Information for DPSCs growing on the FA films).

The morphology of synthetic FA crystals developed from amorphous deposits to hollow balls and finally to
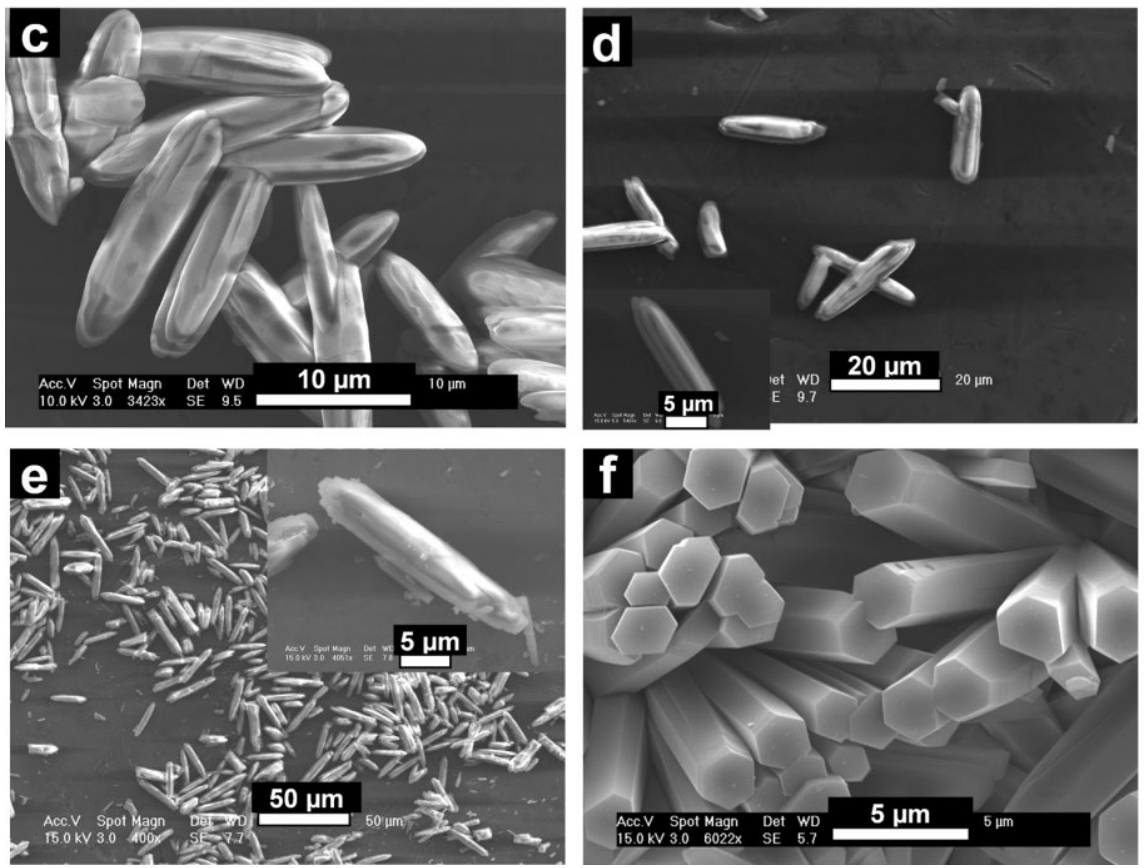

Figure 3. SEM images of samples after autoclaving for $10 \mathrm{~min}$ to $10 \mathrm{~h}$ : a) $10 \mathrm{~min}$; b) $20 \mathrm{~min}$; c) $30 \mathrm{~min}$; d) $1 \mathrm{~h}$; e) $6 \mathrm{~h}$; f) $10 \mathrm{~h}$. As the autoclaving time increased, the crystals changed from shuttlelike structures $(a, b)$ to well-defined microcrystals $(e, f)$. These microcrystals form from FA nanorod bundles, which were created after autoclaving for $6 \mathrm{~h}$ (e). These large units are approximately $20 \mu \mathrm{m}$ in length and $5 \mu \mathrm{m}$ in cross section. Each unit is comprised of a bundle of FA nanorods arranged parallel to each other. These nanorods are approximately $20 \mu \mathrm{m}$ in length and $400 \mathrm{~nm}$ in diameter. The well-defined crystals with a typical apatite hexagonal structure were created after autoclaving for $10 \mathrm{~h}(\mathrm{f})$. well-defined crystals as the autoclave time increased. When the $\mathrm{NaF}$ solution is mixed with EDTA-Ca- $\mathrm{Na}_{2}$ and $\mathrm{NaH}_{2-}$ $\mathrm{PO}_{4}$ at room temperature a suspension is formed after only a few minutes. This is possibly because of the reaction of the $\mathrm{Ca}^{2+}$ which has dissociated from the EDTA-Ca complex with the phosphate and fluoride ions. This may lead to two types of crystals being formed. One is the thermodynamically stable FA $\left[\mathrm{Ca}_{10}\left(\mathrm{PO}_{4}\right)_{6} \mathrm{~F}_{2}\right]$ or a calcium-deficient FA with a rodlike structure that has a hexagonal cross section and a long $c$ axis. ${ }^{[22]}$ The other may be a less thermodynamically stable amorphous fluoridated calcium phosphate (AFCP). This latter compound is kinetically favored at the low temperature and adopts a spherical shape in order to keep its surface energy low. This compound is most likely to form in a solution low in free $\mathrm{Ca}^{2+}$ but with an abundance of phosphate and fluoride ions since $\mathrm{Ca}^{2+}$ ions are strongly chelated by EDTA ligands. As the autoclave time is increased, more $\mathrm{Ca}^{2+}$ ions are dissociated from the EDTA-Ca complex which causes the amorphous AFCP to convert to a more thermodynamically stable FA or cal-

have a very similar appearance to an etched human enamel surface (Fig. 1). Figure 4d shows a similar structure but the enamel prismlike structures, $5-10 \mu \mathrm{m}$ in cross section, more closely approximate the size of human enamel prisms and consist of individual crystals of approximately $100-300 \mathrm{~nm}$ in cross section. We are also able to produce films consisting of crystals of similar size to the human enamel crystals (see Fig. S2 in the Supporting Information).

The FTIR analysis confirms the change to the typical apatite structure over time (see Fig. S3 in the Supporting Information for FTIR spectra of the deposits). In order to further confirm the phase composition and crystallinity of these synthetic crystals, we have conducted powder XRD pattern analyses (see Fig. S4 in the Supporting Information for the XRD data). All of the diffraction peaks can be readily indexed to a cium-deficient FA. Certainly the surface of the spherical AFCP exposed to the solution will react initially with $\mathrm{Ca}^{2+}$ ions released from the EDTA-Ca complex to form FA or calcium-deficient FA multicrystals. The subsequent growth of FA from the continuous outward diffusion of AFCP from the AFCP sphere gives rise to the formation of core-shell or shell structures (Fig. 2b and c), which can be explained by the Kirkendall effect. ${ }^{[23]}$ When the entire AFCP nucleus is dissolved, only the shell-structure is visible (Fig. 2d). Due to the large surface area, these shell structures are not stable, the small multicrystals will dissolve and be redeposited onto the large rodlike FA or calcium-deficient FA crystals due to the Ostwald ripening process. ${ }^{[24]}$ The FA rodlike structures will have the tendency to aggregate together, side by side, to form a bundle because of the stronger van der Waals attraction along 

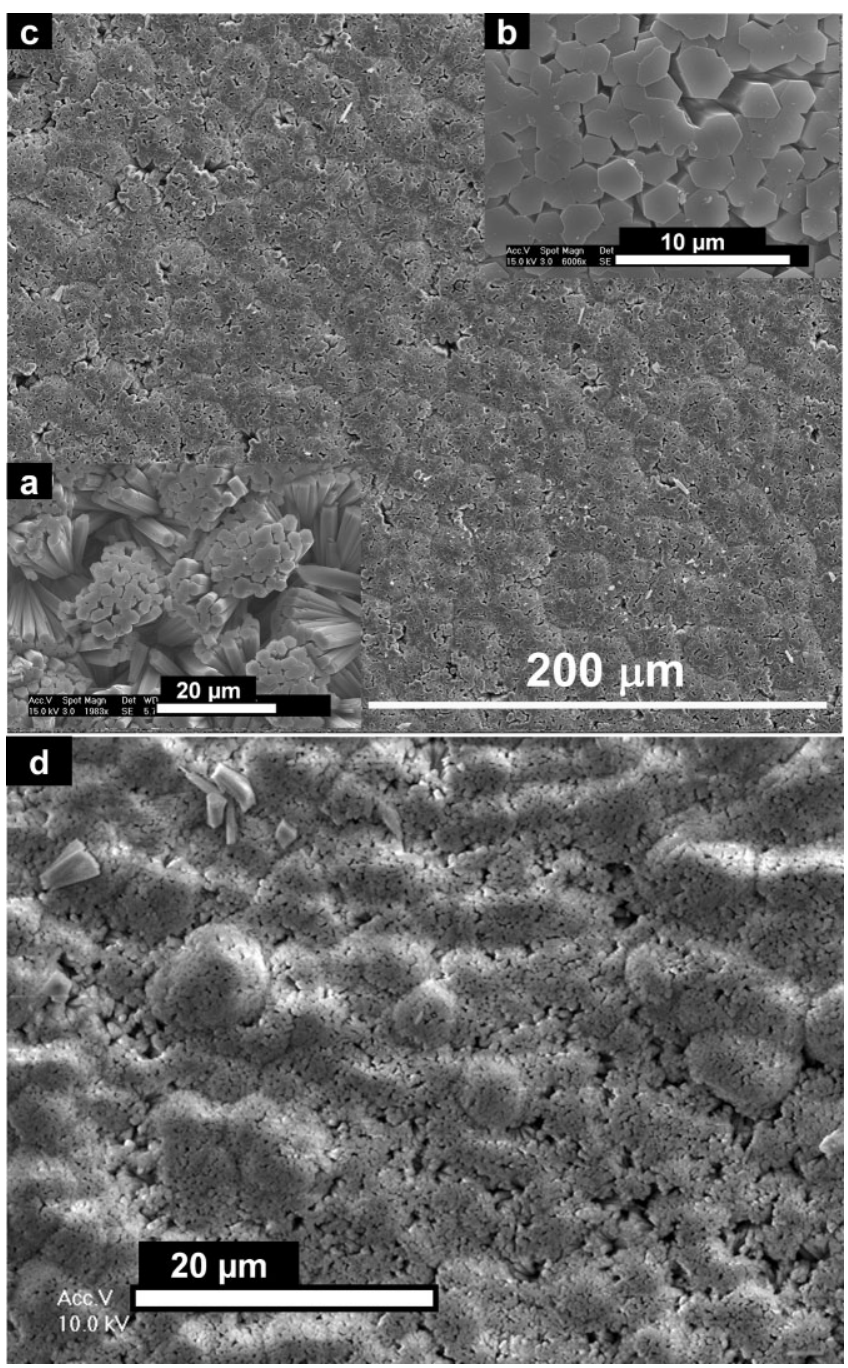

Figure 4. Dental-enamel-like FA films grown on iron plates. The rod-like crystals form bundles (enamel prismlike structures) as shown in (a) and (b) (crystals are 1-3 $\mu \mathrm{m}$ in cross section and approximately $50 \mu \mathrm{m}$ in length, the cross section of the prism structures is about $10-30 \mu \mathrm{m}$ ). c) At lower power the FA crystals that are densely deposited on an iron plate have a very similar appearance to an etched human enamel surface. d) A similar structure to (c) but the enamel prismlike structures, 5-10 $\mu \mathrm{m}$ in cross section, more closely approximate the size of human enamel prisms and consist of individual crystals of approximately 100-300 $\mathrm{nm}$ in cross section.

the long axis of the rods than at the rod ends. This is similar to that described in a recent report of $\mathrm{h}-\mathrm{WO}_{3}$ nanowire growth. ${ }^{[25]}$ A long, large FA nanorod undergoing rapid growth may act as a leader crystal in the center of the bundle while some shorter nanorods become closely aligned parallel to the surface of the leader crystal resulting in a shuttlelike structure. With increasing autoclave time all the $\mathrm{Ca}^{2+}$ precursors from the EDTA-Ca complex are depleted and the bundles stop growing longer. The bundled crystals will fuse to each other and small sharp branch ends with high surface energy will dissolve and tend to fill the kink and steps of the bundle struc- ture to form a large well-defined hexagonal crystal due to the Ostwald ripening process. The whole process of crystal bundle structure evolution is schematically shown in Figure 5.

The large area of well-aligned crystal layers formed on the metal substrate have a similar growth rate and as the crystals grow, the densely packed growth mode along the $c$-axis will force the rodlike crystals to align parallel to each other. Thus, highly orientated FA films are deposited on the substrate. This growth is schematically shown in Figure S6 in the Supporting Information. Moreover, a strong attraction among adjacent rods causes the spontaneous aggregation of rods into bundles (prismlike structures), which have been observed in the cases of varying types of nanorods exhibiting vertical growth on various substrates. ${ }^{[26]}$ Interestingly, these films are very similar to those seen in human enamel and therefore provide a very promising method to create an enamel-like structure. These films can then be used to restore decayed teeth with a material which is similar in chemical composition and structure to the natural tooth enamel. This work also presents experimental evidence as to how the enamel's unique structure is determined. It is reported that the amelogenin is able to self-assemble into microribbons and play a key role in controlling the oriented and elongated growth of apatite crystals during dental enamel development. ${ }^{[8]}$ Our work shows that the unique prism structure of the enamel formation does not need the help of proteins and is able to form due to pure physicochemical interaction of the rodlike apatite crystals. This prismlike structure is stable without the presence of protein. That is why, even though the amelogenin is degraded in the mature enamel, this structure can still persist and remain stable. The proteins may more likely play a key role in the control of the enamel structure during the early development stage.

It should be pointed out that we have tried to deposit the FA films onto different substrates, only the iron-plate substrate is able to produce the enamel-like films. The others may support the growth and orientation of the FA crystals but do not produce the closely aligned prismlike structure (see Fig. S7 in the Supporting Information for FA growing on other substrates). Therefore, the growth of the enamel prismlike structure is not only controlled by the interaction of the crystals but is heavily dependent on the substrate on which it is grown. The reason for this is still under investigation. It is also easier to grow large crystals on these substrates, smaller crystals can be produced, but this depends on the concentration of the solution and the roughness and the chemical composition of the substrate.

The successful growth of DPSCs on these enamel-like FA films not only shows that they are biocompatible, but also shows that under the control of the appropriate signaling molecules and cell-culture conditions it might be possible to induce dentin and pulp formation on the synthetic enamel layer in vitro. Thus, in the future it should be possible to regenerate teeth for implantation into humans. ${ }^{[27]}$

In summary, the present work shows we are able to synthesize both human tooth enamel prismlike structure and enam- 

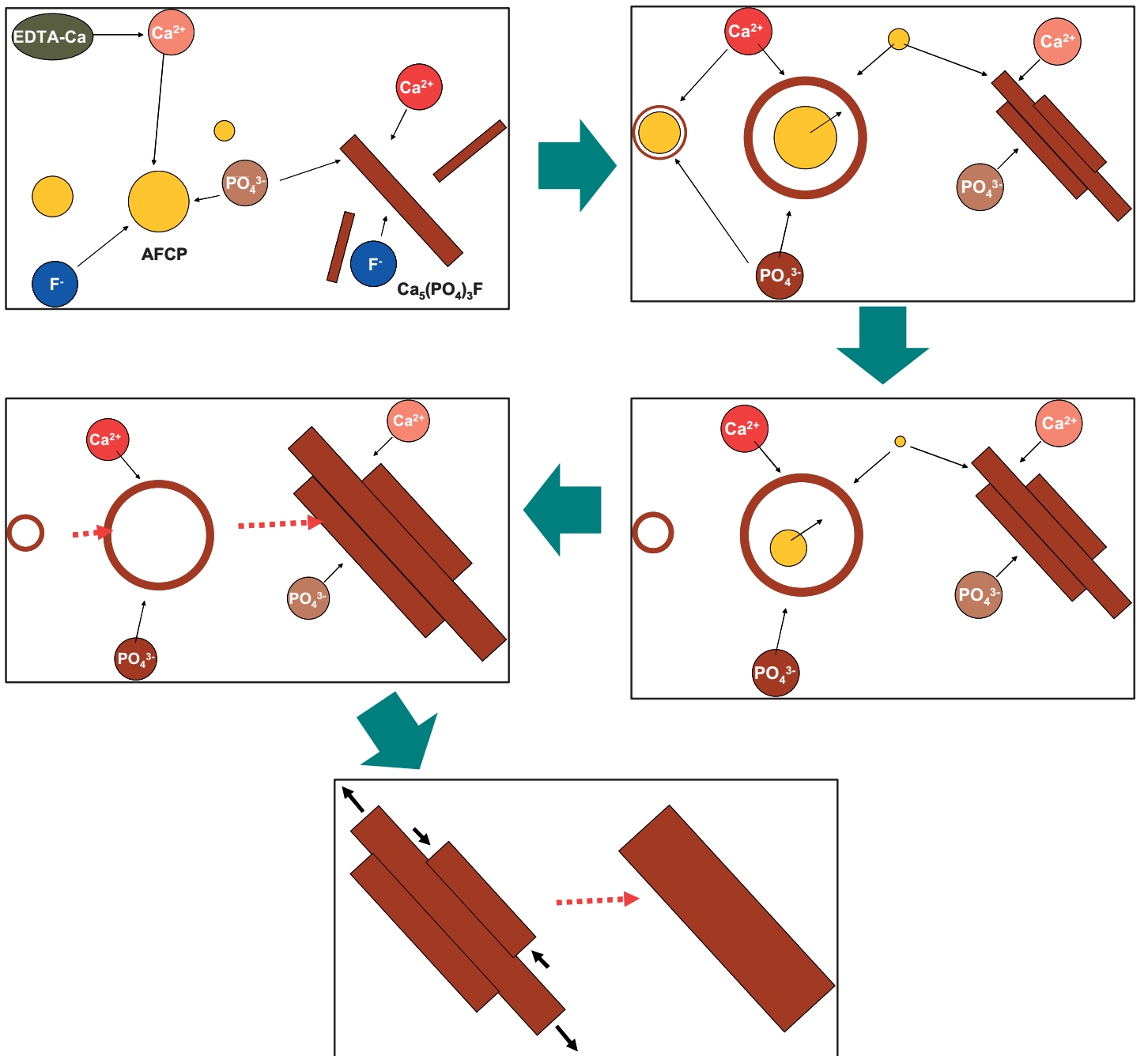

Figure 5. A schematic diagram showing the growth of FA crystals as the autoclave time increased.

el-like films chemically without cells. Both of these structures are very similar in chemical composition and in structural dimensions to natural tooth enamel. Dental pulp stem cells were cultured on these crystal films and showed FA crystals with excellent biocompatibility. This work demonstrates the potential of applying nanotechnology to the direct creation of biomaterials with a specific biological architecture, in this case, human enamel.

\section{Experimental}

Human Enamel Preparation: The natural surface of human enamel was etched lightly (15 s) with $37 \%$ phosphoric acid and its internal profile observed from a longitudinal section; both were imaged with SEM.

Synthesis of FA Crystals: For a typical synthesis: 9.36 g ethylenediaminetetraacetic acid calcium disodium salt (EDTA-Ca-Na $)_{2}$ and $2.07 \mathrm{~g} \mathrm{NaH}_{2} \mathrm{PO}_{4} \cdot \mathrm{H}_{2} \mathrm{O}$ were mixed with about $90 \mathrm{~mL}$ distilled water; the $\mathrm{pH}$ was adjusted to $6.0 ; 0.21 \mathrm{~g} \mathrm{NaF}$ was dissolved in $10 \mathrm{~mL}$ water
( $\mathrm{pH} 7.0)$ and added to the mixture. All the solutions were stirred continuously. The resulting suspensions which contained $0.25 \mathrm{M}$ EDTA$\mathrm{Ca}-\mathrm{Na}_{2}, 0.15 \mathrm{M} \mathrm{NaH} \mathrm{PO}_{4} \cdot \mathrm{H}_{2} \mathrm{O}$ and $0.05 \mathrm{M} \mathrm{NaF}$ were then autoclaved at $121^{\circ} \mathrm{C}$ at a pressure of approximately $2 \mathrm{~atm}\left(1 \mathrm{~atm}=10^{5} \mathrm{~Pa}\right)$ for $5 \mathrm{~min}$ to $10 \mathrm{~h}$. The final suspensions were centrifuged and washed three times with distilled water ( $\mathrm{pH} 7.4)$.

Growth of FA Nanorod Films on Various Substrates: Various substrates (iron, titanium, mica, glass, silicon plates) were added to $100 \mathrm{~mL}$ newly prepared EDTA-Ca- $\mathrm{Na}_{2} / \mathrm{NaH}_{2} \mathrm{PO}_{4} / \mathrm{NaF}$ mixture and then autoclaved at $121^{\circ} \mathrm{C}$ at a pressure of approximately $2 \mathrm{~atm}$ for $10 \mathrm{~h}$. The coated plates were rinsed with distilled water and dried in vacuum before SEM analysis.

Transmission Electron Microscopy: A JEOL 3011 high-resolution electron microscope (JEOL USA, Peabody, MA, USA) operating at $300 \mathrm{kV}$ was used for normal TEM imaging, and high-resolution transmission electron microscopy (HRTEM). The sample was transferred to holey carbon film coated copper grids and dried before TEM analysis.

Scanning Electron Microscopy: SEM analysis and energy-dispersive X-ray spectroscopy analysis were conducted on a Philips XL30FEG scanning electron microscope (FEI company, Hillsboro, OR, USA), FEI Nova Nanolab dual-beam focused ion-beam workstation and scanning electron microscope (FEI company, Hillsboro, OR, USA), 
and JEOL 35C scanning electron microscope (JEOL USA, Peabody, MA, USA) operated at $10-20 \mathrm{kV}$. For better resolution of the SEM image, some of the samples were coated with a $\mathrm{Au} / \mathrm{Pd}$ film.

Received: November 9, 2005

Final version: April 13, 2006

Published online: June 26, 2006

[1] A. Boyde, in Teeth (Eds: B. K. B. Berkovitz, A. Boyde, R. M. Frank, H. J. Hoehling, B. J. Moxham, J. Nalbandian, C. H. Tonge), Handbook of Microscopic Anatomy, Vol.5, Springer, Berlin, Germany 1988, pp. 309-473

[2] Dental Enamel-Formation to Destruction (Eds: C. Robinson, J. Kirkham, R. C. Shore), CRC Press, Boca Raton, FL 1995.

[3] G. Daculsi, J. Mentanteau, L. M. Kerebel, D. Mitre, Calcif. Tissue Int. 1984, 36, 550.

[4] K. Yamagishi, K. Onuma, T. Suzuki, F. Okada, J. Tagami, M. Otsuki, P. Senawangse, Nature 2005, 433, 819.

[5] H. Chen, M. Banaszak Holl, B. Orr, I. Majoros, B. H. Clarkson, J. Dent. Res. 2003, 82, 443.

[6] G. Fincham, J. Moradian-Oldak, J. P. Simmer, J. Struct. Biol. 1999, 126, 270.

[7] C. W. Gibson, Z.-A. Yuan, B. Hall, G. Longenecker, E. Chen, T. Thyagarajan, T. Sreenath, J. T. Wright, S. Decker, R. Piddington, G. Harrison, A. B. Kulkarni, J. Biol. Chem. 2001, 276, 31871.

[8] C. Du, G. Falini, S. Fermani, C. Abbott, J. Moradian-Oldak, Science 2005, 307, 1419.

[9] D. Walsh, J. D. Hopwood, S. Mann, Science 1994, 264, 1576.

[10] J. D. Hartgerink, E. Beniash, S. I. Stupp, Science 2001, 294, 1684.

[11] S. Sadisivan, D. Khushalani, S. Mann, Chem. Mater. 2005, 17, 2765.
[12] E. Fowler, M. Li, S. Mann, H. C. Margolis, J. Mater. Chem. 2005, 15, 3317.

[13] P. Simon, D. Zahn, H. Litchte, R. Kniep, Angew. Chem. Int. Ed. 2006, 45, 1911.

[14] H. Chen, B. H. Clarkson, K. Sun, J. F. Mansfield, J. Colloid Interface Sci. 2005, 288, 97.

[15] T. E. Mallouk, Science 2001, 291, 443.

[16] R. H. Baughman, A. A. Zakhidov, W. A. de Heer, Science 2002, 297, 787.

[17] Y. Xia, P. Yang, Y. Sun, Y. Wu, B. Mayers, B. Gates, Y. Yin, F. Kim, H. Yan, Adv. Mater. 2003, 15, 353.

[18] Y. Fujishiro, A. Fujimoto, T. Sato, A. Okuwaki, J. Colloid Interface Sci. 1995, 173, 119.

[19] W. Suchannek, M. Yoshimura, J. Mater. Res. 1998, 13, 94.

[20] M. Cao, Y. Wang, C. Guo, Y. Qi, C. Hu, Langmuir 2004, $20,4784$.

[21] Mineral Powder Diffraction File Data Book (Eds: P. Bayliss, D. C. Erd, M. E. Mrose, A. P. Sabina, D. K. Smith), JCPDS International Centre for Diffraction Data: Swarthmore, PA 1986. Powder Diffraction File Number: 15-876; pp. 383.

[22] R. Z. LeGeros, Calcium Phosphates in Oral Biology and Medicine, S. Karger AG, Basel, Switzerland 1991.

[23] Y. D. Yin, R. M. Rioux, C. K. Erdonmez, S. Hughes, G. A. Somorjai, A. P. Alivisatos, Science 2004, 304, 711.

[24] A. J. Ardell, V. Ozolins, Nat. Mater. 2005, 4, 309.

[25] Z. J. Gu, Y. Ma, W. S. Yang, G. J. Zhang, J. N. Yao, Chem. Commun. $\mathbf{2 0 0 5}, 3597$.

[26] D. F. Moore, Y. Ding, Z. L. Wang, J. Am. Chem. Soc. 2004, 126, 14372.

[27] Y. Chai, H. C. Slavkin, Microsc. Res. Tech. 2003, 60, 469. 